Artigo original

Hegemonia - Revista Eletrônica do Programa de Mestrado em Direitos Humanos, Cidadania e Violência/Ciência Política do Centro Universitário Unieuro

ISSN: $1809-1261$

UNIEURO, Brasília, número 25 (Especial), 2018, pp. 150-178.

Recebido em: 18/04/2018

Avaliado em: 05/05/2018

Aprovado em: 25/05/2018

\title{
NANOTECNOLOGIA E NANOCIÊNCIA: CONSIDERAÇÕES HISTÓRICA E INTERDISCIPLINAR
}

Cesar Romero Soares Sousa, ${ }^{1}$ Adriana Lima de Holanda, ${ }^{2}$ Anna Beatriz Alves Nunes, ${ }^{3}$ Graziela Silveira Araújo Alves, ${ }^{4}$ e Marina Carvalho Sampaio ${ }^{5}$

Resumo: A nanotecnologia está presente na humanidade acerca de dois mil anos. Inicialmente usada de forma empírica, ao longo dos anos, com o interesse público, seu uso foi racionalizado de maneira sistemática e precisa. Essa ferramenta enquadra tecnologias e processos que manipulam ou exploram estruturas nanométricas em busca de propriedades físico-químicas e comportamentais diferentes daquelas apresentadas em escalas maiores, possibilitando a criação de materiais, substâncias, produtos, dispositivos e sistemas que podem ser aplicados de forma multi e interdisciplinar. Em acelerado e intenso crescimento, a nanotecnologia tem alcançado investimentos vultuosos em todo o mundo, e o Brasil é um dos países que tem investido sua economia nessa tecnologia e, que hoje, possui prioridade de depósito de patentes relacionadas à nanobiotecnologia. Essa ciência inovadora tem permitido significativos avanços em diversas áreas que, não sendo novas, começam a ser reinventadas. Assim, a nanotecnologia está emergindo como a próxima revolução tecnológica, com eventuais efeitos sobre todos os aspectos da vida, sendo que, independente do segmento, há o consenso de que o crescimento da capacidade da construção molecular mudará profundamente o mundo atual.

Palavras chaves: Nanobiotecnologia, Nanométrico e Interdisciplinaridade.

Abstract: Nanotechnology has been around human kind for around two thousand years. It was first used in an empiric way and, then, as public interest grew; its use was rationalized in a precise and systematic way through the years. This tool comprises technologies and processes that

\footnotetext{
${ }^{1}$ Universidade de Brasília (UnB), Brasil, Programa de Engenharia Biomédica. Universidade de Brasília (UNICEUB), Brasília, Brasil.

2 Universidade de Brasília (UNICEUB), Brasília, Brasil.

${ }^{3}$ Universidade de Brasília (UNICEUB), Brasília, Brasil.

${ }^{4}$ Universidade de Brasília (UNICEUB), Brasília, Brasil.

${ }_{5}^{5}$ Programa de Pós-Graduação em Nanociência e Nanobiotecnologia, Universidade de Brasília, Brasil.
} 
Artigo original

Hegemonia - Revista Eletrônica do Programa de Mestrado em Direitos Humanos, Cidadania e Violência/Ciência Política do Centro Universitário Unieuro

ISSN: $1809-1261$

UNIEURO, Brasília, número 25 (Especial), 2018, pp. 150-178.

manipulate or explore nanometric structures in search of physicochemical and behavioral properties that are different from those presented in bigger scales, which makes it possible to create materials, substances, products, devices and systems that can be applied in multi and interdisciplinary ways. Nanotechnology has had fast and intense growth and has reached heavy investments all over the world; Brazil is one of the countries that has invested its economy in this technology, which allows the country to have priority for patent deposits related to nanobiotechnology. This innovative science has allowed meaningful advances in several areas that are not new, but can now be reinvented. Thus, nanotechnology is emerging as the next technological revolution with eventual effects on every aspect of life, and regardless of the segment, there is consensus that the growth in the ability of molecular construction will deeply change the current world.

Keywords: Nanobiotechnology, Nanometric e Interdisciplinarity.

\section{Histórico}

Bem-vindos ao admirável e fascinante mundo muito pequeno e tão grande da nanociência. Os holofotes da ciência há muito destacam e elegem a nanotecnologia e nanobiotecnologia ao patamar de "estado da arte", motivado por sua interdisciplinaridade, com base na química, física, biologia e engenharia de materiais. De modo geral, a motivação para o desenvolvimento da nanociência reside no fato de que as novas e incomuns propriedades físicas e ou químicas que, obviamente, estão ausentes nos mesmos materiais quando em tamanho macro ou micro, são bastantes interessantes, como por exemplo, o seu efeito quântico, no qual aumenta a razão do número de átomos da superfície (maior área de superfície). Assim, proporciona aplicações inovadoras na saúde, como nas áreas biomédicas, de diagnóstico, bioquímica, engenharia, cosmética e beleza, agricultura, nutrição, meio ambiente, além da indústria de microprocessadores, entre outras.

Nanotecnologia pode ser definida como o design intencional para a caracterização, produção e aplicações de materiais, estruturas, dispositivos e sistemas em que pelo menos uma das dimensões está na ordem de grandeza nanométrica. Já a Nanobiotecnologia é um ramo da nanotecnologia voltado às aplicações biológicas e médicas. Neste contexto, os resultados desejáveis da “ação nano" são alcançados com estruturas menores que 100 nanômetros, dentre 
Artigo original

Hegemonia - Revista Eletrônica do Programa de Mestrado em Direitos Humanos, Cidadania e Violência/Ciência Política do Centro Universitário Unieuro

ISSN: $1809-1261$

UNIEURO, Brasília, número 25 (Especial), 2018, pp. 150-178.

os seus efeitos destacam-se: alta biocompatibilidade in vitro e in vivo; capacidade de ultrapassar as barreiras biológicas, como por exemplo, tecidos; invisibilidade ao sistema imune; e maior permanência na circulação sanguínea (LACAVA; MORAIS, 2004; MOGHIMI, 2005; SAHOO; PARVEEN; BAWA, 2007; BRUGIN, 2007; PANDA, 2007; THOMPSON, 2007; CHOUDHURY et al., 2014; SOUSA, 2015; ABDI, 2016; NEVES et al., 2017).

A etimologia nano deriva do prefixo grego nánnos ("excessivamente pequeno”) ou ânos (“anão”). De acordo com o Conselho Nacional de Metrologia, Normalização e Qualidade Industrial - CONMETRO (Resolução no 12, de 12 de outubro de 1988) e definido pela norma ISO/TS 27687:2008, um nanômetro (abreviatura nano) é igual a 10² (0,000 000 001), ou seja, um metro dividido por um bilhão, assim um bilionésimo de metro. Essa medida traduz, portanto, uma molécula de tamanho muito pequeno, sendo que os átomos têm aproximadamente 0,1 a 0,4 nm, assim, em um nanômetro cabem aproximadamente 10 átomos. Em termos de comparações, um vírus tem de 10 a 100 nm (KRUMMENACKER; LEWIS, 1995; COLETI; ALMEIDA, 2010; SAVOLAINEN et al., 2010), e um fio de cabelo humano em média está na ordem de $80.000 \mathrm{~nm}$.

Essencialmente, a nanotecnologia e a habilidade humana de manipular a matéria até o limite do átomo e sintetizar nanomateriais encontra-se entre nós há bastante tempo. Apesar de parecer uma tecnologia recente, é bem mais antiga do que se pode imaginar. Historicamente, é de conhecimento que a tinta nanquim, desenvolvida na China há mais de dois mil anos, é composta de nanopartículas de carvão. E também, ainda no século IV, os romanos produziam pigmentos com partículas coloidais de ouro e prata, encontradas no famoso cálice de Lycurgus (o Cálice de Licurgo) exposto no Museu Britânico, sendo este considerado o mais antigo (fabricado entre os anos de 290 e 325 d.C.) objeto no qual foi utilizado nanomateriais, confeccionado em vidro e impregnado de nanopartículas de ouro e prata (nanopartículas com 50 nanômetros de diâmetro).

Da mesma maneira, nos séculos VI e XV, nos vitrais de algumas das catedrais europeias, teve a presença de nanopartículas de cloreto de ouro e óxido de cloretos metálicos diversos, espalhando diferentes cores. Esta manipulação, a nível molecular, ocorreu de forma não intencional (LIZ-MARZ, 2004; G1, 2013; JACOBI, 2014). No século XIX, o renomado cientista inglês Michael Faraday publicou, em seu artigo intitulado "Experimental relations of gold (and 
Artigo original

Hegemonia - Revista Eletrônica do Programa de Mestrado em Direitos Humanos, Cidadania e Violência/Ciência Política do Centro Universitário Unieuro

ISSN: $1809-1261$

UNIEURO, Brasília, número 25 (Especial), 2018, pp. 150-178.

other metals) to light", que uma das propriedades das nanopartículas de ouro aumentava a absorção da luz emitindo diferentes cores de acordo com o tamanho das nanopartículas. Reportou a formação de soluções coloidais vermelhas de ouro feitas por redução de cloridrato $\left(\mathrm{AuCl}^{-4}\right)$ por fósforo em $\mathrm{CS}^{2}$ (sistema de duas fases) (THOMPSON, 2007; FERREIRA; RANGEL, 2009), sem compreender totalmente ainda estas propriedades, tal qual como os romanos do século IV. No início do século XX, Gustav Mie, físico alemão, fez uma avalição quantitativa relacionada ao fenômeno proposto por Faraday e assumiu que foi o resultado da oscilação coletiva dos elétrons confinados nestas partículas e a homogeneidade das partículas ao seu redor foram descritíveis por suas funções ópticas dielétricas em bulk (MIE, 1908; ALVAREZ et al., 1997; BHARATHI; LEV, 1997).

Em dezembro de 1959, em sua célebre palestra intitulada "There's plenty of room at the bottom" (Há muito espaço lá embaixo) proferida na reunião da Sociedade Americana de Física pelo físico Richard Feynman (o "Pai da Nanotecnologia") ganhador de dois prêmios Nobel, foi considerado o marco inicial da nanotecnologia. A sua proposição foi que, na escala nanométrica, havia muitas coisas a serem reveladas, bem como novas e várias propriedades (POHL; DENK; LANZ, 1984; JACOBI, 2014; DISNER; CESTARI, 2016). Em seguida foi cunhado, em 1974, o termo nanotecnologia pelo pesquisador Norio Taniguchi (TANIGUCHI, 1983).

O olho humano tem um poder de resolução de 1/10 milímetros (100 micrômetros). Assim, se a pessoa olhar para dois pontos ou duas linhas que estão separados por uma distância de menos de 100 micrômetros, ela perceberá apenas um ponto ou uma linha. Como visto, a ideia ou a intuição de manipular e ou alterar o arranjo de átomos para proporcionar ganhos já vem há bastante tempo. Entretanto, o rompimento da barreira da manipulação intencional de objetos de tamanho nanométrico só aconteceu no início dos anos 80, com a invenção de novas técnicas de microscopia de elevada complexidade. Tais microscopias propiciaram o aumento da visão além dos limites do bilionésimo do metro, como por exemplo: microscópio de varredura por tunelamento (Scanning Tunnelling Microscopy, STM), microscópio de força atômica (Atomic Force Microscopy, AFM), microscópio de varredura por sonda (Scanning Probe Microscopy, SPM), microscópio de campo próximo (NFM, near Field Microscopy) (CASTRO, 2002; SENAI, 2004). 
Artigo original

Hegemonia - Revista Eletrônica do Programa de Mestrado em Direitos Humanos, Cidadania e Violência/Ciência Política do Centro Universitário Unieuro

ISSN: $1809-1261$

UNIEURO, Brasília, número 25 (Especial), 2018, pp. 150-178.

Na cronologia moderna, em 1928, surgiu a proposta de Synge, segundo a qual uma nanoesfera metálica (nanopartícula) serviria como fonte evanescente de luz e este princípio levaria a imagens de alta resolução espacial. O advento para o surgimento da geração da primeira imagem espectroscopia de resolução menor que o comprimento de onda da luz, no entanto, só aconteceu em 1984 (SYNGE, 1928; NOVOTNY, 2011).

A nanotecnologia está concentrando investimentos vultuosos no mundo. Países como Estados Unidos (EUA), União Européia (EU) e Japão são os que apresentam o maior nível de desenvolvimento nessa área. Grande parcela do crescimento do mercado de nanotecnologia não provém da produção de nanomateriais básicos, mas sim, da capacidade de alguns segmentos, como o farmacêutico e o de semicondutores, de transformar os nanomateriais básicos em produtos de alto valor agregado (ABDI, 2010).

Os EUA lideram o ranking pelo número de patentes em nanotecnologia. Nas posições seguintes, encontram-se Japão, China, Coréia e Alemanha. As empresas que mais registraram pedidos de patentes foram: IBM, Hewlett-Packard, Mícron Tecnology, AMD, Texas Intruments, Intel e Motorola, Canon, Alcatel-Lucent, Seiko Epson, Sumitomo, Sam-sung, Mitsubish, Toshiba e Procter \& Gamble (CANAVEZ, 2011).

O Brasil, a partir de 2010, aparece em $13^{\circ}$ lugar no ranking de países que tem prioridade para depósito de patentes relacionadas à nanotecnologia e, desde 2008, ocupa essa mesma posição entre aqueles com prioridade para depósito de patentes relacionadas à nanobiotecnologia. Isso demonstra a presença brasileira no desenvolvimento de produtos ou processos relacionados à nanotecnologia e à nanobiotecnologia ou o interesse das firmas estrangeiras no mercado nacional (BATISTA, PEPE; 2014).

O consumo global de todos os tipos de nanomateriais, em 2005, atingiu um volume de 9 milhões de toneladas métricas e US\$13,1 bilhões, com uma expectativa de alcance de 10,3 milhões de toneladas e US\$20,5 bilhões em 2010, considerando-se uma taxa de crescimento anual de 9,3\%, em valor. Materiais orgânicos não-poliméricos respondem pela maior parcela do consumo total e o consumo dos nanomateriais óxidos aumentaram de 8,5\% para 15,75\%, em 2010 (ABDI, 2010).

Os materiais metálicos são o segundo maior segmento com mais de 21\% do mercado. Em 2010, o consumo dos materiais nanoparticulados decresceram 54,6\%, enquanto que os 
Artigo original

Hegemonia - Revista Eletrônica do Programa de Mestrado em Direitos Humanos, Cidadania e Violência/Ciência Política do Centro Universitário Unieuro

ISSN: $1809-1261$

UNIEURO, Brasília, número 25 (Especial), 2018, pp. 150-178.

consumos de filmes, monolíticos e compósitos cresceram a taxas de 25,0\%, 17,4\% e 3,0\%, respectivamente, e em 2012, o mercado foi dominado por semicondutores, mas surgiram novas aplicações baseadas em nanotecnologias para os segmentos farmacêuticos e de saúde, alimentos e outros bens de consumo, devido à estabilidade da cadeia de suprimento dos nanomateriais (ABDI, 2010; PISCOPO et al., 2012).

A comercialização é um ponto marcante na experiência nanotecnológica de países como os Estados Unidos, o Japão, a China, a Índia, os Tigres Asiáticos e os países da Europa. A política de se criar nichos de excelência na nanotecnologia é uma característica marcante da experiência desses países (GOUVEA, 2010). O Brasil vem investindo cada vez mais em nanotecnologia, sendo que estudos relacionados à nanotecnologia vêm sendo incentivados pelo Conselho Nacional de Desenvolvimento Científico e Tecnológico (CNPq) e Ministério de Ciência e Tecnologia (MCT) desde 2001, momento em que foram criadas 4 redes de pesquisa em Nanociência e Nanotecnologia, nas seguintes áreas: materiais nanoestruturados, interfaces e nanotecnologia molecular, nanobiotecnologia e nanodispositivos semicondutores (SANT'ANNA, 2013).

O mercado total de produtos que incorporam nanotecnologias (incluindo semicondutores e eletrônicos) atingiu US\$ 135 bilhões em 2007. Ressalta-se que os valores estimados de mercado não se referem apenas às nanotecnologias incorporadas aos produtos finais, mas sim aos valores dos produtos como um todo. O setor químico é o que ocupa atualmente a maior parcela do mercado global de nanotecnologia, seguido pelos semicondutores. Setores como o farmacêutico, automotivo e defesa representam pequenas parcelas desse mercado. As pesquisas de prospecção tecnológica que vem sendo desenvolvidas no Brasil indicam que as oportunidades de negócio em nanotecnologia tendem a surgir primeiramente nos mercados de cosméticos, produtos provenientes da indústria química (catalisadores, tintas, revestimentos) e petroquímica, plásticos, borrachas e ligas metálicas (ABDI, 2010).

A nanotecnologia tem sido apontada como uma das áreas de grande potencial para atender os objetivos do Milênio das Organizações das Nações Unidas (ONU). O caráter interdisciplinar e multidisciplinar garante a nanotecnologia ser uma tecnologia totalmente inovadora, trazendo benefícios a todas as áreas de estudo (GOMES et al., 2015). 
Artigo original

Hegemonia - Revista Eletrônica do Programa de Mestrado em Direitos Humanos, Cidadania e Violência/Ciência Política do Centro Universitário Unieuro

ISSN: $1809-1261$

UNIEURO, Brasília, número 25 (Especial), 2018, pp. 150-178.

2. Nanotecnologia e Interdisciplinaridade

O desenvolvimento e a aplicação de novas propriedades de materiais e de novos materiais propriamente ditos, tem sido um grande objetivo dos pesquisadores. Vive-se em uma etapa do conhecimento científico-tecnológico no qual a nanotecnologia, em particular, está se tornando presente em todas as áreas da ciência e da tecnologia. Desta forma, os pesquisadores estão investigando maneiras de recorrerem à nanotecnologia em alta escala, determinando suas características a fim de utilizá-las adequadamente, proporcionando novos tipos de materiais (ALMEIDA, 2012).

A nanotecnologia é a tecnologia à escala molecular. Pode-se questionar se essa disciplina não existe já com o nome, bem antigo, de química. Porém, a nanotecnologia, em contraste com a química, procura construir novas moléculas e novos materiais juntando os seus constituintes, átomo a átomo, com uma individualidade e uma precisão que não se consegue quando se trabalha com uma multidão de moléculas. Essa tecnologia tem, de fato, bastante de química, mas também física, biologia, medicina, engenharia eletrotécnica, mecânica, biomédica e química de materiais. É interdisciplinar, uma das marcas da ciência moderna, e as diferentes interfaces são ricas de relevantes problemas científicos e oportunidades de geração de novas tecnologias (DISNER; CESTARI, 2016).

A nanotecnologia tem um significado especial para a inovação industrial. Entre tantas inovações convergentes, a nanociência e a nanotecnologia entram nessa longa aventura como uma alternativa para o estudo dos fenômenos e manipulação de materiais na escala atômica, molecular e macromolecular, quando as propriedades diferem significativamente daquelas observadas na escala macro e a realização do desenho, caracterização, produção de estruturas, peças e sistemas pelo controle do seu tamanho e forma na escala nanométrica, ou $10^{-9}$ (ABDI, 2010; NNI, 2000).

As nanopartículas possuem propriedades mecânicas, óticas e químicas específicas e de grande aplicabilidade para os setores químico, têxtil, automotivo, citando apenas alguns exemplos. Alguns produtos finais que resultam da nanotecnologia incluem tecidos que não 
Artigo original

Hegemonia - Revista Eletrônica do Programa de Mestrado em Direitos Humanos, Cidadania e Violência/Ciência Política do Centro Universitário Unieuro

ISSN: $1809-1261$

UNIEURO, Brasília, número 25 (Especial), 2018, pp. 150-178.

molham, polímeros resistentes tanto quanto o aço e o alumínio, películas para vidros e cosméticos cujas partículas penetram nos poros da pele, entre outros (PISCOPO et al., 2014).

Considerada um dos principais focos das atividades de pesquisa, a nanotecnologia é, na atualidade, desenvolvimento e inovação em todos os países industrializados. Suas consequências trazem enormes avanços ao bem-estar material das sociedades, principalmente pela maior eficácia da aplicabilidade devido às propriedades físicas e químicas dos materiais nanoestruturados. Entretanto, para tornar possível o surgimento de outras aplicações tecnológicas, ainda existem diversos aspectos científicos a serem explorados. Sendo assim, o estudo de nanoestruturas é de extrema importância tanto do ponto de vista científico quanto tecnológico (PISCOPO et al., 2014).

De acordo com a Agência Brasileira de Desenvolvimento Industrial (ABDI, 2010, BATISTA; PEPE, 2014). Dentre as possíveis aplicações da nanotecnologia presentes em diversas áreas, destacam-se:

a) Indústria automobilística e aeronáutica - Fabricação de materiais mais leves, pneus mais duráveis, plásticos não inflamáveis e mais baratos.

b) Indústria farmacêutica, biotecnológica e biomédica - Criação de novos medicamentos baseados em nanoestruturas, de materiais para regeneração de ossos e tecidos, de sensores para glicose, $\mathrm{CO}_{2}$ e colesterol.

c) Indústria química e de materiais - Produção de catalizadores mais eficientes, ferramentas de corte mais duras, fluidos magnéticos inteligentes.

d) Indústria eletrônica e de comunicações - Armazenamento de dados, em telas planas, aumento da velocidade de processamento.

e) Meio ambiente - Desenvolvimento de membranas seletivas para remover contaminantes ou sal da água, novas possibilidades de reciclagem, fabricação de nanocatalizadores mais eficazes e econômicos para conversão catalítica em escapamentos automotivos, fabricação de nanosensores para detecção de agentes tóxicos e vazamentos, fabricação de nanodispositivos para separação de gases, desenvolvimento de nanomembranas para purificação da água, dessalinização e desintoxicação, de nanossensores para detecção de contaminantes e agentes patogênicos, entre outros. 
Artigo original

Hegemonia - Revista Eletrônica do Programa de Mestrado em Direitos Humanos, Cidadania e Violência/Ciência Política do Centro Universitário Unieuro

ISSN: $1809-1261$

UNIEURO, Brasília, número 25 (Especial), 2018, pp. 150-178.

f) Produtividade agrícola - Desenvolvimento de zeólitas nanoporosas para liberação lenta e controlada de água, de fertilizantes e agrodefensivos, de nanocápsulas liberadoras de herbicidas, de nanossensores para monitoração do solo e do desenvolvimento de plantas, de nanopartículas magnéticas para combater a contaminação do solo, produção de nanossensores para detecção de pragas, nanopartículas transportadoras ou liberadoras de pesticidas, inseticidas, ou de ação repelente.

g) Setor energético - Economia de energia ao utilizar materiais mais leves e circuitos menores, fabricação de células fotovoltaicas e fotoeletroquímicas, fabricação de dispositivos orgânicos emissores de luz, aplicações de nanotubos de carbono em células solares, fabricação de nanocatalizadores para produção de hidrogênio, produção de células a combustível, fabricação de nanomateriais para armazenamento de hidrogênio.

h) Setor de fabricação - Desenvolvimento de novos microscópios e instrumentos de medida, de ferramentas para manipular a matéria a nível atômico, de bioestruturas, etc.

\subsection{Tipos de nanopartículas}

Há diversos modelos diferentes de nanopartículas. Desde as sintetizadas pelo homem até aquelas provenientes da natureza e de fenômenos químicos como a combustão de combustível, por exemplo. A fumaça preta que comumente é vista saindo de ônibus e caminhões é formada por uma gama de nanopartículas (SILVA; VIANA; MOHALLEM, 2009). As nanopartículas produzidas artificialmente podem ser classificadas como lipossomas, nanoemulsões, nanopartículas lipídicas sólidas, nanopartículas poliméricas, dentre inúmeros outros tipos. Os lipossomas e as nanoemulsões são particularmente importantes pois, são nanoestruturas aplicadas no estudo do projeto Rapha para o tratamento de feridas no pé diabético.

\subsubsection{Lipossomas}

Lipossomas são nanopartículas que possuem uma ou mais bicamadas fosfolipídicas. Tais camadas se dispõem em torno de um compartimento aquoso e têm o objetivo de carrear 
Artigo original

Hegemonia - Revista Eletrônica do Programa de Mestrado em Direitos Humanos, Cidadania e Violência/Ciência Política do Centro Universitário Unieuro

ISSN: $1809-1261$

UNIEURO, Brasília, número 25 (Especial), 2018, pp. 150-178.

fármacos, biomoléculas ou compostos utilizados em diagnósticos. A estabilidade pode variar de acordo com fatores biológicos, físicos e químicos. Por apresentarem camada(s) lipídica(s) e compartimento aquoso, são capazes de carrear substâncias tanto hidrofílicas quanto lipofílicas (BATISTA; CARVALHO; MAGALHÃES, 2007).

Tais nanopartículas são empregadas desde 1980 em infecções fúngicas sistêmicas e para o tratamento do câncer. Também são usadas na produção de vacinas, na terapia gênica, em doenças parasitárias e cardíacas (BATISTA; CARVALHO; MAGALHÃES, 2007).

\subsubsection{Nanoemulsões}

Nanoemulsões são estruturas coloidais formadas pela dispersão de dois líquidos imiscíveis e cineticamente estáveis (BRUXEL et al., 2012; SAMPAIO, 2017). Podem ser dispersões tanto óleo em água $(\mathrm{O} / \mathrm{A})$ quanto água em óleo $(\mathrm{A} / \mathrm{O})$. As nanoemulsões mais comuns são O/A, facilitam a solubilidade de substâncias hidrofóbicas e, assim, a biodisponibilidade de tais compostos é aprimorada (GANTA; AMIJI, 2009; GIANELLA et al., 2011; RAGELLE et al., 2012; ZHANG et al., 2013; BU et al., 2014; CHOUDHURY et al., 2014; GANTA et al., 2015; FOFARIA et al., 2016; SAMPAIO, 2017). Há cerca de 40 anos, as nanoemulsões têm sido empregadas como fonte de calorias e ácidos graxos. Podem ser carreadoras de fármacos como benzodiazepínicos, anestésicos e corticoides (BRUXEL et al., 2012).

\subsubsection{Nanopartículas lipídicas sólidas}

As nanopartículas lipídicas sólidas são sistemas coloidais que atuam como agentes encapsuladores, protetores e distribuidores de substâncias lipofílicas. Assim como as nanoemulsões, há a presença de uma fase aquosa e de uma oleosa. A diferença entre os dois sistemas é que o núcleo lipídico das nanopartículas das nanoemulsões é líquido, enquanto que o centro lipídico das nanopartículas lipídicas é sólido. As fases de uma nanopartícula lipídica sólida são estabilizadas por um ou mais emulsificantes (ASSIS et al., 2012). 
Artigo original

Hegemonia - Revista Eletrônica do Programa de Mestrado em Direitos Humanos, Cidadania e Violência/Ciência Política do Centro Universitário Unieuro

ISSN: $1809-1261$

UNIEURO, Brasília, número 25 (Especial), 2018, pp. 150-178.

Tais nanopartículas são especialmente empregadas na indústria alimentícia. Vitaminas e nutracêuticos como $\beta$-caroteno, licopeno, luteína e fitoesteróis são encapsulados nas nanopartículas lipídicas (WEISS et al., 2008; ASSIS et al., 2012).

\subsubsection{Nanopartículas poliméricas}

As nanopartículas poliméricas compreendem as nanoesferas e nanocápsulas, as quais diferem entre si quanto à composição e à organização estrutural. Estas são formadas por um invólucro de polímeros que circunda o núcleo oleoso. Já as nanoesferas apresentam uma matriz polimérica e não possuem óleo em sua composição. São estruturas com diâmetro menor que $1000 \mathrm{~nm}$ (SCHAFFAZICK et al., 2003).

$\mathrm{Na}$ área da saúde são amplamente empregadas como medicamentos de administração parenteral, oral ou oftálmica. São sistemas bastante estudados para carrearem fármacos anticancerígenos e antibióticos (SCHAFFAZICK et al., 2003).

\subsection{Nanotecnologia e Saúde: diagnóstico, tratamentos, nutrição, estética e cosmetologia}

Uma das áreas mais promissora das aplicações da nanotecnologia é a saúde, devido seu impacto direto na vida dos seres humanos. Já são inúmeras pesquisas e uma grande variedade de produtos nanotecnológicos aplicados às melhorias no âmbito da prática clínica, principalmente nos setores da imaginologia médica e da indústria farmacêutica. Com um alavancar tão abrupto nas últimas décadas, hoje já se fala sobre a nanomedicina, a ciência que utiliza ferramentas nano para aprimorar recursos na saúde, envolvendo tratamento, diagnóstico, monitoramento, prevenção e controle de doenças, engenharia para a recuperação de tecidos, criação de cosméticos e terapia nutricional (FIGUEIRAS; COIMBRA; VEIGA, 2014; ALENCAR; BOCHNER; ANTUNES, 2017). 
Artigo original

Hegemonia - Revista Eletrônica do Programa de Mestrado em Direitos Humanos, Cidadania e Violência/Ciência Política do Centro Universitário Unieuro

ISSN: $1809-1261$

UNIEURO, Brasília, número 25 (Especial), 2018, pp. 150-178.

\subsubsection{Diagnóstico}

$\mathrm{Na}$ nova era, em que a otimização de recursos, tempo, e espaço são conceitos essenciais e almejados por um mercado cada vez mais digital e tecnológico, a nanotecnologia foi a ferramenta ideal para possibilitar a criação de aparelhos laboratoriais com menores dimensões, mais ágeis, e com resultados precisos e fidedignos, sem, entretanto, necessitarem de um manuseamento especializado (SAHOO, 2012). Assim, os nanosensores ou biossensores permitem um diagnóstico ultrarrápido e extremamente sensível e específico através de aparelhos compactos que reconhecem e sinalizam a presença, atividade ou concentração mínima de um analito (metabólitos específicos, ácidos nucleicos, proteínas, patógenos e células) utilizando nanopartículas como pontos quânticos. Como vantagem adicional, esses sensores utilizam métodos de medição baratos e diretos, levando a uma produção de baixo custo e sendo facilmente transportáveis, além de permitirem a medição de mais de mais de um analito simultaneamente (BOULAIZ et al., 2011; CHOI, 2012).

Acredita-se que no futuro próximo, os biossensores pessoais já estejam disponíveis para indivíduos que necessitem monitorar continuamente seus níveis sanguíneos por motivo de distúrbio metabólico, gerando praticidade e conforto ao paciente. De uma forma mais inovadora ainda, a tecnologia diminuta e precisa desses aparelhos abriram a prerrogativa para que dispositivos implantáveis sejam introduzidos no organismo fazendo, além da análise da concentração de certas substâncias, a liberação automática do fármaco desejado. Os sensores injetáveis impedem uma resposta imunológica devido ao tamanho reduzido dos nanossistemas, tendo assim, uma maior duração que outros dispositivos tradicionais. Contudo, essa abordagem terapêutica necessita de pesquisas que comprovem sua real eficácia e aceitação em pacientes (CASH; CLARK, 2011).

Os biossensores ainda podem ser utilizados para a finalidade de analisar a constituição genética de um indivíduo de forma rápida e precisa, neste caso, são os chamados biossensores de DNA, compostos por uma sonda de DNA ligada à superfície de um transdutor eletroquímico capaz de revelar a predisposição genética para certas doenças e firmar o diagnóstico para doenças hereditárias. Esse encontro da Nanomedicina com a Genômica tende a ser um campo 
Artigo original

Hegemonia - Revista Eletrônica do Programa de Mestrado em Direitos Humanos, Cidadania e Violência/Ciência Política do Centro Universitário Unieuro

ISSN: $1809-1261$

UNIEURO, Brasília, número 25 (Especial), 2018, pp. 150-178.

revolucionário para prevenção e detecção precoce de patologias (FIGUEIRAS; COIMBRA; VEIGA, 2014).

A prática da nanotecnologia na imaginologia trouxe uma excepcional revolução para a saúde, principalmente se tratando de doenças neoplásicas, pois possibilitou o amplo acompanhamento de processos de metástase, mesmo que iniciais, além da detecção da doença em estágio mais precoce, fator determinante para obtenção de um prognóstico favorável. O uso de nanossistemas também possibilitou a formulação de agentes de contraste com intensidade de fluorescência adequada e isentos da fotodegração, além de mais seguros em comparação aos contrastes da imaginologia convencional, que apresentam um forte poder iatrogênico (BOULAIZ et al., 2011).

No ramo da imaginologia ótica, com aplicação de nanopartículas quantum dots (QDs) moléculas inorgânicas semicondutoras que emitem fluorescência quando iluminadas por luz ultravioleta - foi possível observar vantagens em relação à imaginologia ótica convencional. Essas partículas são resistentes à fotodegradação e degradação química, permitindo maior durabilidade e intensidade da reação. A área de ressonância magnética (RM) também mostra resultados satisfatórios nas pesquisas com o emprego de nanopartículas como agentes de contraste com alta capacidade resolutiva para imagens dos rins e cérebro, e tecidos com células neoplásicas, ou com pequenas metástases, sendo que na RM tradicional, cânceres precoces e metastização inicial não eram percebidos na imagem (MURTHY, 2007).

\subsubsection{Tratamento de doenças - entrega e liberação de fármacos}

Inúmeros estudos buscam avaliar nanoformulações para o desenvolvimento de fármacos que melhorem a eficácia terapêutica, dando origem à nanofármaco, que não se restringe à criação de novas substâncias farmacológicas como também possibilita a reformulação e, consequente, recuperação de fármacos promissores que haviam sido inviabilizados para a comercialização devido taxa de efeitos colaterais, toxicidade, rápida metabolização, baixa biodisponibilidade e falta de seletividade. As indústrias farmacêuticas têm investido exponencialmente na pesquisa e no desenvolvimento de novos medicamentos nanotecnológicos com o objetivo de produzir 
Artigo original

Hegemonia - Revista Eletrônica do Programa de Mestrado em Direitos Humanos, Cidadania e Violência/Ciência Política do Centro Universitário Unieuro

ISSN: $1809-1261$

UNIEURO, Brasília, número 25 (Especial), 2018, pp. 150-178.

compostos com maior biocompatibilidade, solubilidade e estabilidade em relação aos fármacos convencionais (BATISTA; PEPE, 2014).

Como peça chave nessa questão, encontra-se as nanoformulações com o objetivo de melhorar a entrega e liberação dos fármacos, restringindo-os ao sítio específico para diminuir a toxidade sistêmica e efeitos colaterais indesejáveis (TOMITAKA et al., 2009; WILCZEWSKA, 2012). A menor quantidade do princípio ativo a ser administrada, gera um menor custo para produção, além de menor período de administração que pode refletir sobre uma melhor aceitação do tratamento pelo paciente, ponto essencial para sucesso terapêutico. Já são inúmeros sistemas nanoestruturados (lipossomas, nanopartículas poliméricas, nanotubos de carbono, nanopartículas metálicas ou dendrímeros) sendo avaliados em estudos pré-clínicos e clínicos para carregamento e liberação de uma substância, que vai desde fármacos, vacinas, proteínas recombinantes e oligonucleotídeos (PARVEEN; MISRA; SAHOO, 2012; GHARPURE et al., 2015)

Na prática clínica, os lipossomas foram os primeiros nanofármacos a serem empregados com aprovação para administração endovenosa. A incorporação do fármaco ao lipossoma ocorre através de um mecanismo de encapsulamento, no qual o fármaco será liberado lentamente, fator que prolonga seu tempo de duração e ação sobre tecidos. Contudo, alguns fatores estão envolvidos nesta liberação, como: composição do lipossoma, do gradiente osmótico, do $\mathrm{pH}$ e do ambiente circundante, o que deve ser analisado para evitar respostas inadequadas (WILCZEWSKA et al., 2012; FIGUEIRAS; COIMBRA; VEIGA, 2014). Os primeiros fármacos lipossomais foram: doxorrubicina conjugada com polietilenoglicol (PEG) (Doxil@), introduzida em 1995 na clínica para tratamento do sarcoma de Kaposi em pacientes com HIV positivo, com resultados superiores em relação ao aumento da biodisponibilidade do fármaco convencional (REYNOLDS et al., 2012; GHARPURE et al., 2015); o Myocet e o Daunoxome que foram utilizados para tratamento de câncer, com grande vantagem da redução de toxicidade cardíaca; e a Anfotericina B, introduzida no mercado em 1998, com significativa redução em sua toxicidade renal, para utilização clínica no tratamento da leishmaniose visceral e de micoses (STYLIANOPOULOS; JAIN, 2015).

As nanopartículas poliméricas tem apresentado vantagens em comparação aos lipossomas devido possuírem maior estabilidade e durabilidade e menor custo. Na prática clínica, 
Artigo original

Hegemonia - Revista Eletrônica do Programa de Mestrado em Direitos Humanos, Cidadania e Violência/Ciência Política do Centro Universitário Unieuro

ISSN: $1809-1261$

UNIEURO, Brasília, número 25 (Especial), 2018, pp. 150-178.

as nanopartículas poliméricas são usadas em próteses ortopédicas e fios de suturas biodegradáveis, e, atualmente, tem despertado interesse em relação ao seu uso como carreadores de DNA para a terapia gênica. Para situações que tem como objetivo a liberação de substâncias de forma mais lenta, como no caso de vacinas ou drogas para tratamento de doenças crônicas, tem-se utilizado nanopartículas poliméricas com tamanho superior a 1000nm (BATISTA; PEPE, 2014).

Em estudos clínicos, essas nanopartículas poliméricas tem sido empregue para melhorar a biodisponibilidade de drogas convencionais, aumentando sua meia-vida plasmática, diminuindo os efeitos colaterais e aumentando sua ação em sítios alvo, como foi o caso do Paclitaxel em formulação de nanopartículas de albumina (Abraxane®) e do Paclitaxel poliglumex em formulação polimérica com ácido L-glutâmico (MIELE et al., 2009; GHARPURE et al., 2015; STYLIANOPOULOS; JAIN, 2015).

As nanopartículas inteligentes são partículas padronizadas para um direcionamento alvoespecífico, diminuindo os efeitos colaterais sistêmicos. Assim, associa-se o fármaco a um nanoveículo que irá direcionar a partícula ao lugar a ser liberada (WILCZEWSKA et al., 2012; SAHOO, 2012). Uma forma de gerar essa vetorização das partículas é através da inclusão de substâncias magnéticas com a posterior aplicação exógena de um campo magnético sobre o local a ser liberado o fármaco. As nanopartículas magnéticas são muito promissoras por serem biocompatíveis, com excelente estabilidade química e física e bons carreadores, gerando também um menor risco de toxicidade, menor tempo de circulação no organismo, com controle adequado da dose, local de ação e tempo de eliminação (MATTEI et al., 2009; GUGLIELMO et al., 2010; CÓTICA et al., 2012; XU; SUN, 2013).

Contudo, essa nova terapêutica pode ter seus limitantes, como o tempo em que as partículas permanecem ligadas às células-alvos pode ser curto, pois as células realizam a endocitose de forma automática de substâncias presas à sua membrana citoplasmática, além disso, a velocidade do fluxo sanguíneo e a intensidade do campo magnético influenciarão a manutenção da partícula magnética no local desejado, devendo estes fatores serem observados e padronizados para viabilidade da prática clínica (FONTANIVE et al., 2014). 
Artigo original

Hegemonia - Revista Eletrônica do Programa de Mestrado em Direitos Humanos, Cidadania e Violência/Ciência Política do Centro Universitário Unieuro

ISSN: $1809-1261$

UNIEURO, Brasília, número 25 (Especial), 2018, pp. 150-178.

2.2.3 Engenharia biomédica: regeneração de órgãos e tecidos, construção de próteses

Outra aplicação da Nanomedicina, envolve criação de nanomateriais para reconstrução de tecidos, órgãos e implantes (MURA; COUVREUR, 2012). Muitos biomateriais têm sido explorados como arcabouços tridimensionais para a regeneração tecidual, sendo que esses devem ser biocompatíveis para prevenir o processo de rejeição imunológica e formarem a estrutura ideal para fixação e proliferação de células, permitindo sua expressão proteica normal. Neste contexto, os hidrogéis peptídicos tem se mostrado ideais como estruturas moleculares, permitindo a fixação das células e também a difusão irrestrita dos fatores de crescimento e sinais celulares para o processo regenerativo, além de, contribuírem para a angiogênese, servindo como agente de entrega de fatores terapêuticos, como fator básico de crescimento de fibroblastos e fator de crescimento endotelial vascular (LI et al., 2013; CHAN; ZHUO; NI, 2015). Para aumentar a longevidade e a biocompatibilidade de dispositivos cirúrgicos e próteses tem sido usado nanocompósitos de liga de titânio (CHAN et al., 2017).

Estudos mostram o sucesso para implantes de células nervosas crescidas em malhas poliméricas para reparo de medula espinhal; células ósseas ou de cartilagem para reconstituição de articulações e células hepáticas para construção de fígado para transplante (CHAN et al., 2017). Lipossomas sintéticos foram apresentados como um agente hemostático infusível para melhorar o estímulo da ativação plaquetária (OKAMURA et al., 2009), tendo a sua biodistribuição e propriedades hemostáticas caracterizadas em vários modelos animais préclínicos (HAGISAWA et al., 2015). Também em modelo experimental, foi mostrado o uso de nanopartículas hemostáticas baseadas em polímeros sintéticos biocompatíveis (PGLA) e revestidas com um peptídeo para redução do tempo de sangramento (BERTRAM et al., 2009) e para melhorar a sobrevida após lesão blástica em cânceres sanguíneos (LASHOF-SULLIVAN et al., 2014; DESBOROUGH et al., 2016).

\subsubsection{Estética e cosmetologia}

$\mathrm{Na}$ esfera estética, a Nanotecnologia surge como estratégia para potencializar a permeação de princípios ativos contidos nos cosméticos. Através de nanoestruturas os ativos 
Artigo original

Hegemonia - Revista Eletrônica do Programa de Mestrado em Direitos Humanos, Cidadania e Violência/Ciência Política do Centro Universitário Unieuro

ISSN: $1809-1261$

UNIEURO, Brasília, número 25 (Especial), 2018, pp. 150-178.

são encapsulados para liberação controlada na pele, tendo assim uma melhor aplicação tátilsensorial e maior poder de penetração dos produtos, atingindo locais nos quais os produtos convencionais não conseguem alcançar. No caso de maquiagens, essa tecnologia possibilitou a produção de cores diferenciadas e mais irradiantes. Pesquisas na área da cosmética abrangem estudos avaliando: protetores solares, produtos antienvelhecimento, produtos emagrecedores, hidratantes para a pele e cabelo e também maquiagens diversas (PAPAKOSTAS et al., 2011).

Apesar de inúmeros estudos nessa área mostrando sucesso nas pesquisas pré-clínicas, também há relatos que indicam a indesejável presença de efeitos sistêmicos, como a possibilidade de penetração dos nanossistemas nos pulmões; ou sua distribuição pelo sistema linfático, podendo atingir até mesmo, o Sistema Nervoso Central (HOUGEIR; KIRCIK, 2012).

\subsubsection{Nutrição e nanotecnologia}

A utilização da nanotecnologia relacionada à nutrição é, na verdade, bem ampla. Muitos componentes nutricionais bioativos poderão ser disponibilizados através dessa tecnologia, sendo que nanossistemas circulantes no organismo poderão liberar alimentos em locais previamente programados. Ressalta-se ainda, a possibilidade de através desse recurso entender complexas interações biomoleculares, podendo com isto aumentar a precisão da terapia nutricional, monitorando em tempo real a dietoterapia, bem como controle de suas consequências sistêmicas. Pacientes com disfagia aguardam ansiosamente avanços terapêuticos como esse, a fim de suprir sua carência nutricional, levando-os à preservação ou reabilitação orgânica, e prevenção de efeitos secundários à deficiência alimentar (WEISS; TAKHISTOV; CLEMENTS, 2006).

Por outro lado, os nanossensores podem ser utilizados como embalagens para proteção e detecção de microrganismos e/ou toxinas presentes nos alimentos e estão entre as fronteiras mais promissoras no âmbito da nanociência da nutrição (WEISS, TAKHISTOV; CLEMENTS, 2006; CHUN, 2009).

Acredita-se, atualmente, que a nanotecnologia é uma das ferramentas mais importantes para o setor agroalimentar porque pode contribuir para a melhoria da produtividade dos alimentos. Há uma expectativa de que o uso dessa tecnologia possa contribuir para melhorar o 
Artigo original

Hegemonia - Revista Eletrônica do Programa de Mestrado em Direitos Humanos, Cidadania e Violência/Ciência Política do Centro Universitário Unieuro

ISSN: $1809-1261$

UNIEURO, Brasília, número 25 (Especial), 2018, pp. 150-178.

rendimento das culturas, sem danificar o solo e a água, e reduzir a perda de nitrogênio provocada por lixiviação e emissões. Outros benefícios estão relacionados ao desenvolvimento de espécies resistentes aos insetos e ao processamento e armazenamento com aumento de vida útil do alimento por meio da utilização de embalagens inteligentes (RESCH; FARINA, 2015).

\section{Políticas Públicas da Nanotecnologia}

A Política Nacional de Incentivo à Ciência, Tecnologia e Inovação (PNCTI) é administrada pelo governo e o incentivo específico na área de nanotecnologia foi uma iniciativa do Ministério da Ciência, Tecnologia e Inovação (MCTI) (TENÓRIO; MELLO; VIANA, 2017).

As principais políticas públicas em vigor para a nanotecnologia são a criação dos Institutos Nacionais de Incentivo a Ciência e Tecnologia (INCTs), a Iniciativa Brasileira em Nanotecnologia (IBN) e a criação do Sistema Nacional de laboratórios em Nanotecnologia (SISNano) (ABDI, 2016). Outro integrante marcante da PNCTI é a Política Nacional de Ciência, Tecnologia e Incentivo à Saúde (PNCTIS). Regidos pelos mesmos princípios, a PNCTI e a PNCTIS, buscam o desenvolvimento da tecnologia com importância social (BRASIL; MINISTÉRIO DA SAÚDE; SECRETARIA DE CIÊNCIA TECNOLOGIA E INSUMOS ESTRATÉGICOS, 2008).

Os INCTs foram instituídos pelo Ministério da Ciência, Tecnologia e Inovação pela portaria MCT n $\mathrm{n}^{\mathrm{4}}$ 429, de 17 de julho de 2008 e são concentrados em áreas específicas de conhecimento, com foco na nanotecnologia direcionada para saúde. Seu objetivo é incitar e reunir, de maneira planejada, grupos de pesquisa, destacando o desenvolvimento de nanomedicamentos na área da saúde. O incentivo a pesquisa básica e aplicada promove o desenvolvimento científico e tecnológico com eixo na integração entre o Sistema Brasileiro de Tecnologia (Sibratec) e a indústria. A nanotecnologia voltada para a saúde é separada em nove INCTs apresentadas na tabela 1. Cada uma apresenta um nível de organização e tem como propósito a formação de recursos humanos e transferência de conhecimento para a sociedade (ABDI, 2016; CNPQ; MINISTÉRIO DA CIÊNCIA, 2013). 
Artigo original

Hegemonia - Revista Eletrônica do Programa de Mestrado em Direitos Humanos, Cidadania e Violência/Ciência Política do Centro Universitário Unieuro

ISSN: $1809-1261$

UNIEURO, Brasília, número 25 (Especial), 2018, pp. 150-178.

Tabela 1-Institutos Nacionais de Ciência e Tecnologia nas Áreas de Nanotecnologia aplicada à Saúde

\begin{tabular}{ll} 
Sigla & Instituto Nacional de Ciência e Tecnologia: Nanotecnologia e Saúde \\
\hline INOMAT & INCT em Materiais Complexos Funcionais \\
INCTMN & INCT dos Materiais em Nanotecnologia \\
NanoBioSimes & INCT de NanoBioEstruturas e Simulação Biomolecular \\
NanoBiofar & INCT de Nanobiofarmacêutica \\
\hline Nanobiotecnologia & INCT de Nanobiotecnologia \\
INCTIF & INCT para Inovaçăo Farmacêutica \\
\hline INCTV & INCT de Vacinas \\
\hline Nanobiosimes & INCT de NanoBioEstruturas e Simulação NanoBioMolecular \\
\hline INCTCatálise & INCT de Catálise em Sistemas Moleculares Nanoestruturados \\
\hline Inomat & INCT em Materiais Complexos Funcionais \\
\hline NAMITEC & INCT de Sustenas Nucri e Babieketrônicos (NAMITEC) \\
\hline- & INCT de Nanomateriais de Carbono \\
\hline DISSE & INCT de Nanotecnologia para Marcadores Integrados \\
\hline - & INCT de Nanodispostivos Semicondutores (DISSE) \\
\hline N-BIOFAR & INCT de Nanobiotecnologia \\
\hline INCT-INOFAR & INCT de Nanobiofarmacêutica (N-BIOFAR) \\
\hline- & INCT de Fármacos e Medicamentos (INCT-INOFAR) \\
\hline BIOFABRIS & INCT em Tuberculose \\
\hline
\end{tabular}

(ABDI, 2016).

A IBN, lançada em 2013 pelo MCTI, foi elaborada com intuito de fomentar o mercado econômico em nanotecnologia e abrir caminhos para que o Brasil alcance reconhecimento. A função da IBN é planejar, elaborar, executar, acompanhar e avaliar as atividades desempenhadas em nanotecnologia pela Estratégia Nacional de Ciência, Tecnologia e Inovação (ENCITI). O acompanhamento, análise e sugestões de políticas para a nanotecnologia é realizada por uma das coordenações da Secretaria de Desenvolvimento Tecnológico e Inovação (SETEC) do MCTI, a Coordenação Geral de Micro e Nanotecnologia (CGNT), que conta com um Comitê Consultivo de Nanotecnologia (CCNano), estruturado por 12 membros de setores públicos e privado. O Sistema Nacional de laboratórios em Nanotecnologia (SISNano) foi produto da IBN. Este soma uma rede de 26 laboratórios que voltam seus investimentos para a infraestrutura, o que permite 
Artigo original

Hegemonia - Revista Eletrônica do Programa de Mestrado em Direitos Humanos, Cidadania e Violência/Ciência Política do Centro Universitário Unieuro

ISSN: $1809-1261$

UNIEURO, Brasília, número 25 (Especial), 2018, pp. 150-178.

alavancar a pesquisa e o desenvolvimento dessa tecnologia. Através de editais de subvenção, a IBN promove o financiamento de projetos inovadores realizados nessa área (ABDI, 2016).

A PNCTIS, assim como a PNCTI, tem como propósito o avanço tecnológico de modo sustentável, que promova uma coesão social e alavanque o mercado através da integração entre as academias e indústrias (BRASIL; MINISTÉRIO DA SAÚDE; SECRETARIA DE CIÊNCIA TECNOLOGIA E INSUMOS ESTRATÉGICOS, 2008; FONSECA, 2016). De modo específico, a Financiadora de Estudos e Projetos (FINEP), é responsável por intermediar o contato entre as empresas, universidades e institutos científicos e tecnológicos com o intuito de valorizar o desenvolvimento de uma economia sustentável (OECD, 2005).

Muito tem se debatido a respeito dos impactos do desenvolvimento tecnológico em âmbito social, econômico e na comunidade acadêmica. Mesmo com as políticas de incentivo, o processo que designa a verba para as pesquisas até então, mostra-se pouco compatível com as diretrizes. Entretanto, para os estudos que ainda chegam a ser realizados e publicados, é levantada uma outra questão: qual a relevância dos estudos e sua aplicabilidade real? (TRENTIN et al., 2018). As políticas públicas e os órgãos que auxiliam na regulamentação e incentivo à ciência estão buscando essa conjunção a fim de diminuir os resultados negativos.

\section{Considerações Finais}

Será o fascinante mundo da nanotecnologia uma das maiores forças impulsionadoras para o que hoje já se fala "terceira revolução industrial”? É fato que sim! Uma ferramenta que tem mudado o mundo, com novos produtos e materiais, impactando significativamente a sociedade e a vida do ser humano. E isto ainda é só o princípio.

A nanotecnologia é um campo interdisciplinar emergente, com alto poder para revolucionar cada área onde seja explorada e utilizada. No entanto, muitas de suas aplicações ainda se encontram em fase de desenvolvimento, e outras por surgir. Contudo, a expectativa já foi lançada e a projeção é que num futuro próximo essa tecnologia assuma um papel preponderante nos avanços e conquistas do desenvolvimento técnico-científico e tecnológico. 
Artigo original

Hegemonia - Revista Eletrônica do Programa de Mestrado em Direitos Humanos, Cidadania e Violência/Ciência Política do Centro Universitário Unieuro

ISSN: $1809-1261$

UNIEURO, Brasília, número 25 (Especial), 2018, pp. 150-178.

Referências Bibliográficas

ABDI - Agência Brasileira de Desenvolvimento Industrial, "Cartilha Sobre Nanotecnologia", Cartilha Sobre Nanotecnologia, 2010, 60 pp.

ABDI - Agência Brasileira de Desenvolvimento Industrial, “Agenda Tecnológica Setorial - ATS Complexo Industrial da Saúde Nanotecnologia Panorama Econômico”, 2016, 86 pp.

ALENCAR, M.S.M.; BOCHNER, R.; DIAS, M.F.F.; ANTUNES, A.M.S.A. "Análise da produção científica brasileira sobre nanotecnologia e saúde”, In Reciis - Rev Eletron Comun Inf Inov Saúde, vol. 11, 2017, num. 1, 31 de março de 2017, 1-16 pp.

ALMEIDA, C. C. C. F., "Prospecção Tecnológica a Médio e Longo Prazo Sobre o Desenvolvimento e Aplicações da Nanotecnologia em Materiais Poliméricos”, Campinas, Universidade de Campinas, 2012, 128 pp.

ALVAREZ, M. M. et al. “Optical Absorption Spectra of Nanocrystal Gold Molecules”. In The Journal of Physical Chemistry B, vol. 101, 1997 n 19, 04 de março de 1997, 3706-3712 pp.

ASSIS, L. M. et al., "Características de nanopartículas e potenciais aplicações em alimentos” In Brazilian Journal of Food Technology, vol. 15, 2012, num. 2, Campinas, junho de 2012, 99-109 pp.

BATISTA, A. J. S.; PEPE, V. L. E. "Os desafios da nanotecnologia para a vigilância sanitária de medicamentos”. In Ciência \& Saúde Coletiva, vol. 19, 2014, num. 7, 2105-2114 pp.

BAWA, R., "Patents and nanomedicine”, In Nanomedicine, vol. 2, 2007, num. 3, Londres, Reino Unido, 08 de junho de 2007, 351-374 pp.

BAtista, C. M., CARVALHO, C. M. B., MAGALHÃES, N. S. S., "Lipossomas e suas aplicações terapêuticas: Estado da arte”, In Revista Brasileira de Ciências Farmacêuticas, vol. 43, 2007, num. 2, abril a junho de 2007, 167-179 pp.

BERTRAM, J.P.; WILLIAMS, C.A.; ROBINSON, R.; SEGAL, S.S.; FLYNN, N.T.; LAVIK, E.B. "Intravenous hemostat: nanotechnology to halt bleeding.", In Science Translational Medicine, vol. 1, 2009, num.11, 16 de dezembro de 2009, 11-22 pp.

BRUXEL, F. et al., "Nanoemulsões como sistemas de liberação parenteral de fármacos”, In Química Nova, vol. 35, 2012, num. 9, 1827-1840 pp. 
Artigo original

Hegemonia - Revista Eletrônica do Programa de Mestrado em Direitos Humanos, Cidadania e Violência/Ciência Política do Centro Universitário Unieuro

ISSN: $1809-1261$

UNIEURO, Brasília, número 25 (Especial), 2018, pp. 150-178.

Brasil. Ministério da Saúde. Secretaria de Ciência, Tecnologia e Insumos Estratégicos. Departamento de Ciência e Tecnologia, Política nacional de ciência, tecnologia e inovação em saúde, 2 edição, Brasília, Editora do Ministério da Saúde, Série B. Textos básicos em saúde, 2008, 44 pp.

Brasil. "INCT - Institutos Nacionais de Ciência e Tecnologia", Brasília, Ministério da Ciência, Tecnologia e Inovação, 2013, 291 pp.

BOULAIZ, H.; ALVAREZ, P. J.; RAMIREZ, A.; MARCHAL, J. A.; PRADOS, J.; RODRIGUEZSERRANO, F.; PÉRAN, M.; MELGUIZO, C.; ARANEGA, A. "Nanomedicine: application areas and development prospects", In International Journal of Molecular Sciences, vol. 12, 2011, num. 5, 19 de maio de 2011, 3303-3321 pp.

BU, H. et al., “A TPGS-incorporating nanoemulsion of paclitaxel circumvents drug resistance in breast cancer", In International Journal of Pharmaceutics, vol. 471, 2014, num. 1 e 2, 206-213 pp.

CANAVEZ, M. J. M., “O Uso da Nanotecnologia nas Empresa: Um Estudo de Caso no Setor de Cosméticos”, Curitiba, Universidade Federal do Paraná, 2011, 81 pp.

CASH, K. J.; CLARK, H. A. "Nanosensors and nanomaterials for monitoring glucose in diabetes”, In Trends in Molecular Medicine, vol. 16, 2010, num. 12, 23 de setembro de 2010, 584-593 pp.

CHAN, K.H.; ZHUO, S.; NI, M. "Priming the surface of orthopedic implants for osteoblast attachment in bone tissue engineering. ", In Int Journal Med Science, vol. 12, 2015, num. 9, 14 de agosto de 2015, 701-707 pp.

CHAN, K.H.; LEE, W.H.; ZHUO, S.; NI, M. "Harnessing supramolecular peptide nanotechnology in biomedical applications. ", In International Journal of Nanomedicine, vol. 12, 2017, 9 de fevereiro de 2017, 1171-1182 pp.

CHOI, C. "Integrated nanobiosensor technology for biomedical application", In Nanobiosensors in Disease Diagnosis, vol. 1, 2012, num. 1, 16 de fevereiro de 2012, 1-4 pp. CHOUDHURY, H. et al. "Improvement of cellular uptake, in vitro antitumor activity and sustained release profile with increased bioavailability from a nanoemulsion platform.", In International Journal of Pharmaceutics, vol. 460, 2013, num. 1-2, 02 de janeiro de 2014, 131$143 \mathrm{pp}$. 
Artigo original

Hegemonia - Revista Eletrônica do Programa de Mestrado em Direitos Humanos, Cidadania e Violência/Ciência Política do Centro Universitário Unieuro

ISSN: $1809-1261$

UNIEURO, Brasília, número 25 (Especial), 2018, pp. 150-178.

CHUN, A. L. "Will the public swallow nanofood?”, In Nature Nanotechnology, vol. 4, 2009, num. 12, 790-801 pp.

COLETI, J. S.; MARIA, G.; ALMEIDA, D. B. "Aspectos morfológicos da terminologia da nanociência e nanotecnologia", In Filologia e Linguística Portuguesa, vol. 12, 2010, num. 2, 2010, 271-294 pp.

CÓTICA, L.F.; FREITAS, V.F.; DIAS, G.S.; SANTOS, I.A.; KHALIL, N.M., et al. "Simple and facile approach to synthesize magnetite nanoparticles and assessment of their effects on blood cells.”, In J Magn Magn Mater, vol. 324, 2011, num.4, fevereiro de 2012, 559-563 pp.

DESBOROUGH, M.J.R.; SMETHURST, P.A.; ESTCOURT, L.J.; STANWORTH, S.J. “Alternatives to allogeneic platelet transfusion”. In British Journal of Haematology, vol. 175, 2016, num. 3, 21 de setembro de 2016, 381-392 pp.

DISNER, G. R.; CESTARI, M. M., "Nanociência \& Nanotecnologia”. In Evidência, vol. 16, 2016, num. 1, Joaçaba, janeiro a junho de 2016, 71-76 pp.

FERREIRA, H. S.; RANGEL, M. C. "Nanotecnologia: aspectos gerais e potencial de aplicação em catálise”, In Química Nova, v. 32, 2009, num. 7, 04 de agosto de 2009, 1860-1870 pp.

FIGUEIRAS, A.R.R.; COIMBRA, A.B.; VEIGA, F.J.B. "Nanotecnologia na saúde: aplicações e perspectiva”, In Boletim Informativo Geum, vol. 5, 2014, num. 2, abr./jun. de 2014, 14-26 pp. FOFARIA, N, M. et al., "Nanoemulsion formulations for anti-cancer agent piplartine characterization, toxicological, pharmacokinetics and efficacy studies", In International Journal of Pharmaceutics, vol. 498, 2015, num. 1 e 2, 10 de fevereiro de 2016, 12-22 pp.

FONSECA, Paulo FC. "Políticas Públicas de Ciência, Tecnologia e Inovação: possíveis contribuições para uma governança responsável da nanotecnologia no Brasil”, In Nanotecnologias, Desenvolvimento e Meio ambiente. Revista do Programa de Pós-Graduação em Sociologia / Programa de Pós-Graduação em Sociologia, Universidade Federal de Sergipe, vol. 2016, num. 29, São Cristovão, jul./dez., 83-120 pp.

FONTANIVE, V.C.P.; KHALIL, N.M.; COTICA, L.F.; MAINARDES, R.M. “Aspectos físicos e biológicos de nanopartículas de ferritas magnéticas.”, In Rev Ciênc Farm Básica Aplicada, vol. 35, 2014, num. 4, 14 de outubro de 2013, 549-558 pp.

G1. Taça de 1. 600 anos que muda de cor já usava princípios de nanotecnologia Objeto apresenta cores diferentes conforme a posição da fonte de luz . 2013. Disponível em: 
Artigo original

Hegemonia - Revista Eletrônica do Programa de Mestrado em Direitos Humanos, Cidadania e Violência/Ciência Política do Centro Universitário Unieuro

ISSN: 1809-1261

UNIEURO, Brasília, número 25 (Especial), 2018, pp. 150-178.

<http://g1.globo.com/ciencia-e-saude/noticia/2013/08/taca-de-1600-anos-que-muda-de-

cor-ja-usava-principios-de-nanotecnologia.html>. Acesso em: 01/abr./18.

GANTA, S., AMIJI, M., "Coadministration of Paclitaxel and Curcumin in Nanoemulsion

Formulations To Overcome Multidrug Resistance in Tumor Cells”, In Molecular Pharmaceutics, vol. 6, 2009, num. 3, 03 de novembro de 2009, 928-939 pp.

GANTA, S. et al., "EGFR Targeted Theranostic Nanoemulsion for Image-Guided Ovarian Cancer Therapy", In Pharmaceutical Research, vol. 32, 2015, num. 8, 04 de março de 2015, 27532763 pp.

GHARPURE, K.M.; WU, S.Y.; LI C.; LOPEZ-BERESTEIN, G. and ANIL K. "Nanotechnology: Future of Oncotherapy", In Clin Cancer Res., vol.21, 2015, num.14, 15 de julho de 2015, 3121-3130 pp.

GIANELLA, A. et al., "Multifunctional Nanoemulsion Platform for Imaging Guided Therapy Evaluated in Experimental Cancer”, In ACS NANO, vol. 5, 2011, num. 6, 4422-4433 pp.

GOMES, R. C. et al., "Aplicações da nanotecnologia na indústria de alimentos. Uma Revisão", In Revista Brasileira de Higiene e Sanidade Animal, vol. 9, 2015, num. 1, 28 de março de 2015, 1-8 pp.

GOUVEA, R., “Nanotecnologia, Um Novo Paradigma de Desenvolvimento Economico: Uma Análise da Experiência Internacional \& Brasileira”, In Estratégica, vol. 9, 2010, num. 8, junho de 2010, 46-67 pp.

GUGLIELMO, C.D.; LOPEZ, D.R.; LAPUENTE, J.; MALLAFRE, J.M.L.; SUÀREZ, M.B. "Embryotoxicity of cobalt ferrite and gold nanoparticles: A first in vitro approach." In Reprod Toxicol, vol.30, 2010, num.2, setembro de 2010; 271-276 pp.

HAGISAWA, K.; NISHIKAWA, K.; YANAGAWA, R.; KINOSHITA, M.; DOI, M.; SUZUKI, H.; IWAYA, K.; SAITOH, D.; SEKI, S.; TAKEOKA, S.; HANDA, M.; NISHIDA, Y. "Treatment with fibrinogen gamma-chain peptide-coated, adenosine 5'diphosphateencapsulated liposomes as an infusible hemostatic agent against active liver bleeding in rabbits with acute thrombocytopenia.", In Transfusion, vol. 55, 2015, num. 2, 22 de agosto de 2014, 314-325 pp.

HOUGEIR, F. G.; KIRCIK, L. “A review of delivery systems in cosmetics.”, In Dermatologic Therapy., vol. 25, 2012, num. 3, 22 de agosto de 2012, 234-237 pp. 
Artigo original

Hegemonia - Revista Eletrônica do Programa de Mestrado em Direitos Humanos, Cidadania e Violência/Ciência Política do Centro Universitário Unieuro

ISSN: $1809-1261$

UNIEURO, Brasília, número 25 (Especial), 2018, pp. 150-178.

JACOBI, M. M., "El admirable mundo Nano: Nanociencia y Nanotecnología" In SLTCAUCHO, 2014, num. 4, 8-15 pp.

KRUMMENACKER, M.; LEWIS, J., Prospects in Nanotechnology: Toward Molecular Manufacturing, 1 ed., Wiley, 1995, 297 pp.

LACAVA, Z. G. M.; MORAIS, P. C., "Aplicações biomédicas de nanopartículas magnéticas.", In Parcerias Estratégicas, vol. 9, 2004, num. 18, agosto de 2004, 73-86 pp.

LASHOF-SUllivAN, M.M., SHOFFSTALL, E., ATKINS, K.T., KEANE, N., BIR, C., VANDEVORD, P. \& LAVIK, E.B. "Intravenously administered nanoparticles increase survival following blast trauma.", In Proceedings of the National Acadamy of Sciences of the United States of America, vol. 111, 2014, num. 28, 30 de julho de 2014, 10293-10298 pp.

LI, X.; WANG L.; FAN, Y.; FENG, Q.; CUI, F-Z.; WATARI F. "Nanostructured scaffolds for bone tissue engineering.", In Journal Biomed Materials Research Part A., vol. 101, 2013, num. 8, fevereiro de 2013, 2424-2435 pp.

MATTEI ,Y.C.; PEREZ, O.P. "Synthesis of high-coercivity cobalt ferrite nanocrystals." In Microelectron Journal., vol. 40, 2009, num. 4-5, abril de 2009, 673-676 pp.

MIELE E, SPINELLI GP, MIELE E, TOMAO F, TOMAO S. “Albumin-bound formulation of paclitaxel (Abraxane $(\mathbb{R})$ ABI-007) in the treatment of breast cancer", In Int Journal Nanomedicine, vol.4, 2009; 20 de abril de 2009, 99-105 pp.

MOGHIMI, S. M.; HUNTER, A. C.; MURRAY, J. C., "Nanomedicine: current status and future prospects.", In The FASEB Journal, vol. 19, 2005, num. 3, 01 de maio de 2005, 311-330 pp.

MURA, S.; COUVREUR, P. “Nanotheranostics for personalized medicine.”, In Advanced Drug Delivery Reviews, vol. 64, 2012, num. 13, outubro de 2012, 1394-1416 pp.

MURTHY, S. K. "Nanoparticles in modern medicine: state of the art and future challenges", In International Journal of Nanomedicine, vol. 2, 2007, num. 2, 129-141pp.

NEVES, W. P. et al."Comparative Efficacy of a Biocompatible Citrate-Functionalized Magnetic Fluid Mediating Radiofrequency Hyperthermia and Magnetohyperthermia to Treat Ectopic Ehrlich-Solid-Tumor-Bearing Elderly Mice." In Journal of Cancer Science \& Therapy, vol. 9, 2017, num. 4, 10 de abril de 2017, 393-400 pp.

NOVOTNY, L., "From near-field optics to optical antenas”, In Physics Today, vol. 64, 2011, 
Artigo original

Hegemonia - Revista Eletrônica do Programa de Mestrado em Direitos Humanos, Cidadania e Violência/Ciência Política do Centro Universitário Unieuro

ISSN: $1809-1261$

UNIEURO, Brasília, número 25 (Especial), 2018, pp. 150-178.

n. 7, 47-52 pp.

OECD - Organização para a cooperação e desenvolvimento econômico, "Manual de Oslo", FINEP - Financiadora de Estudos e Projetos, terceira edição, 2005.

OKAMURA, Y.; TAKEOKA, S.; ETO, K.; MAEKAWA, I.; FUJIE, T.; MARUYAMA, H.; IKEDA, Y.; HANDA, M. "Development of fibrinogen gamma-chain peptide-coated, adenosine diphosphate-encapsulated liposomes as a synthetic platelet substitute.", In Journal of Thrombosis and Haemostasis, vol. 7, 2009, num. 3, março de 2009, 470- 477 pp.

PAPAKOSTA, D; RANCAN, F.; STERRY, W; BLUME-PEYTAVI, U.; VOGT, A. "Nanoparticles in dermatology.", In Dermatological Research, vol. 303, 2011, num. 8, agosto de 2011, 533-550 pp.

PARVEEN S., MISRA R., SAHOO S.K. "Nanoparticles: a boon to drug delivery, therapeutics, diagnostics and imaging", In Nanomedicine, vol.8, 2011, num.2, fevereiro de 2012, 147-166 pp. PISCOPO, M. R. et al. “O setor brasileiro de nanotecnologia: Oportunidades e desafios”, In Revista de Negócios, vol. 19, 2014, num. 4, 11 de novembro de 2014, 43-63 pp.

POHL, D. W.; DENK, W.; LANZ, M. “Optical stethoscopy: Image recording with resolution ג/20”. In Applied Physics Letters, vol. 44, 1984, num. 7, 12 de janeiro de 1984, 651-653 pp.

RAGELLE, H. et al., "Nanoemulsion formulation of fisetin improves bioavailability and antitumour activity in mice", In International Journal of Pharmaceutics, vol. 427, 2012, num. 2, 24 de fevereiro de 2012, 452-459 pp.

RESCH, S.; FARINA, M. C. "Mapa do Conhecimento em Nanotecnologia no setor agroalimentar”. In rev. adm. mackenzie, vol.16, 2015, num. 3, 16 de Março de 2015, 51-75 p.p. REYNOLDS, J.G.; GERETTI, E.; HENDRIKS, B.S.; LEE, H.; LEONARD, S.C.; KLINZ, S.G.; et al. "HER2-targeted liposomal doxorubicin displays enhanced anti-tumorigenic effects without associated cardiotoxicity", In Toxicology and Applied Pharmacology, vol. 262, 2012, num.1, julho de 2012, 1-10 pp.

SCHAFFAZICK, S. R., "Caracterização e estabilidade físico-química de sistemas poliméricos nanoparticulados para administração de fármacos”, In Química Nova, vol. 26, 2003, num. 5, 726-737 pp.

SAHOO, S. K.; PARVEEN, S.; PANDA, J. J., "The present and future of nanotechnology in human health care." In Nanomedicine: Nanotechnology, Biology, and Medicine, vol. 3, 2007, 
Artigo original

Hegemonia - Revista Eletrônica do Programa de Mestrado em Direitos Humanos, Cidadania e Violência/Ciência Política do Centro Universitário Unieuro

ISSN: $1809-1261$

UNIEURO, Brasília, número 25 (Especial), 2018, pp. 150-178.

num. 1, março de 2007, 20-31 pp.

SAHOO, S.K. "Nanotechnology in Health Care", ebook, vol.1, Singapure, Pan Stanford Publishing: United Kingdom, , 2012, 20 p.

SAMPAIO, M. C., "Desenvolvimento e caracterização de nanoemulsões à base de óleo de buriti (Mauritia flexuosa) para avaliação de efeitos biológicos em células de câncer de mama in vitro", Brasília, Universidade de Brasília, 2017, 87 pp.

SANT'ANNA, L. S., “Patenteamento em nanotecnologia no Brasil: desenvolvimento, potencialidades e reflexões para o meio ambiente e a saúde pública”, Rio de Janeiro, Fundação Oswaldo Cruz, 2013, 209 pp.

SAVOLAINEN, K. et al. "Risk assessment of engineered nanomaterials and nanotechnologiesA review". In Toxicology, v. 269, 2010, num. 2-3, 25 de janeiro de 2010, 92-104 pp.

SEKHON, B. S., "Nanotechnology in agrifood production: an overview”. In Nanotechnology, Science and Applications, vol.7, 2014, num. 31-53, 20 de maio de 2014, 23 p.p.

SOUSA, C. R. S., "Citrato E Nanocápsulas Contendo Selol No Ehrlich Ortotópico Em Camundongo Swiss.", Brasília, Brasil, Universidade de Brasília, 2015, 1-147 pp.

SILVA, S. L. A., VIANA, M. M., MOHALLEM, N. D. S., "Afinal, o que é Nanociência e Nanotecnologia? Uma Abordagem para o Ensino Médio”, In Química Nova na Escola, vol. 31, 2009, num. 3, 172-178 pp.

STYLIANOPOULOS, T.; JAIN, R.K. "Design considerations for nanotherapeutics in oncology", In Nanomedicine: Nanotechnology, Biology, and Medicine, 22 de julho de 2015, 1 55 pp.

SYNGE, E., "A suggested method for extending microscopic resolution into the ultramicroscopic region", In The London, Edinburgh, and Dublin Philosophical Magazine and Journal of Science, vol. 6, 1928, num. 35, 356-362 pp.

TANIGUCHI, N., "Current Status in, and Future Trends of, Ultraprecision Machining and Ultrafine Materials Processing.” In CIRP Annals - Manufacturing Technology, vol. 32, 1983, n 2, 573-582 pp.

TENÓRIO, Marge et al. "Políticas de fomento à ciência, tecnologia e inovação em saúde no Brasil e o lugar da pesquisa clínica”, In Ciência \& Saúde Coletiva, vol. 22, 2017, num. 5, 1441 1454 pp. 
Artigo original

Hegemonia - Revista Eletrônica do Programa de Mestrado em Direitos Humanos, Cidadania e Violência/Ciência Política do Centro Universitário Unieuro

ISSN: $1809-1261$

UNIEURO, Brasília, número 25 (Especial), 2018, pp. 150-178.

TOMITAKA A, HIRUKAWA A, YAMADA T, MORISHITA S, TAKEMURA Y. "Biocompatibility of various ferrite nanoparticles evaluated by in vitro cytotoxicity assays using HeLa cells", In Journal Magn Magn Mater, vol. 321, maio de 2009, num.10, 2009, 1482-1484 pp. THOMPSON, D. "Michael Faraday's recognition of ruby gold: the birth of modern nanotechnology." In Gold Bulletin, vol. 40, 2007, dezembro de 2007, num. 4, 267-269 pp. TRENTIN, Eliana Claudia Pinto; ROCHA, Israel Lobato; DA SILVA, Marcília Martins, “O avanço da pesquisa científica e qualificação dos cientistas brasileiros”, In Artigos e comunicações, Multi-Science Journal, vol. 1, 2018, num. 10, Goiás, janeiro de 2018, 9-11 pp. WEISS, J.; TAKHISTOV, P.; CLEMENTS, J. C. "Functional materials in food nanotechnology.”, In Journal of Food Science, vol. 71, 2006, num. 9, 13 de novembro de 2006, 107-116 pp.

WILCZEWSKA, A. Z; NIEMIROWICZ, K.; MARKIEWICZ, K. H.; CAR, H. “Nanoparticles as drug delivery systems", In Pharmacological Reports, vol. 64, 2012, num. 5, 22 de maio de 2012, 1020-1037 pp.

XU, C.; SUN, S. "New forms of superparamagnetic nanoparticles for biomedical applications.", In Review Article. Adv Drug Deliv Reviews, vol.65, 2013, num.5, maio de 2013, 732-743 pp. ZHANG, Z. et al., "A self-assembled nanocarrier loading teniposide improves the oral delivery and drug concentration in tumor", In Journal of Controlled Release, vol. 166, 2013, 20 de dezembro de 2012, 30-37 pp. 